EPJ Web of Conferences 66, 03053 (2014)

DOI: $10.1051 /$ epjconf/ 20146603053

(C) Owned by the authors, published by EDP Sciences, 2014

\title{
Core excitations in the structure and reactions of halo nuclei
}

\author{
J. A. Lay ${ }^{1,2, a}$, A. M. Moro², J. M. Arias², and J. Gómez-Camacho ${ }^{2,3}$ \\ ${ }^{1}$ Dipartimento di Fisica e Astronomia "Galileo Galilei", Università di Padova and INFN, Sezione di Padova, \\ I-35131, Padova, Italy \\ ${ }^{2}$ Departamento de FAMN, Universidad de Sevilla, Apdo. 1065, E-41080, Sevilla, Spain \\ ${ }^{3}$ Centro Nacional de Aceleradores (U. Sevilla, J. Andalucía, CSIC), Av. Thomas A. Edison, E-41092 Sevilla, \\ Spain
}

\begin{abstract}
We analyse the resonant break-up of halo nuclei including possible excitations of the core both in the structure of the halo nucleus and in the reaction dynamics. The structure of the halo nucleus is treated in the weak-coupling limit, assuming a particle+core model with core excitations. The bound and resonant states of this system are calculated as an expansion in a pseudo-state basis, for which we use a recent generalization of the Transformed Harmonic Oscillator basis. This basis is used within a distorted wave Born approximation reaction framework which includes possible excitations and deexcitations of the core during the collision. We apply this scheme to describe the resonant break-up of ${ }^{11} \mathrm{Be}$ on ${ }^{12} \mathrm{C}$ at $67 \mathrm{MeV} / \mathrm{u}$. Comparing with the available experimental data from RIKEN [N. Fukuda et al., PRC 70, 054606 (2004)] we discuss the possibility of extracting spectroscopic information of ${ }^{11} \mathrm{Be}$ resonances from the analysis of the break-up angular distributions.
\end{abstract}

\section{Introduction}

The study of reactions involving loosely bound exotic nuclei has been one of the most active fields in Nuclear Physics during the last few years. This kind of reactions is known to be strongly influenced by the coupling to the unbound states of the weakly bound nucleus. Therefore, there has been a great interest in finding a realistic description of the continuum of these nuclei.

In reaction calculations, one-neutron halo nuclei are usually described using a two-body model, comprising an inert core and one valence particle. This inert-core model can be inadequate in situations where the states of the core nucleus contain significant admixtures of core excited components. Furthermore, recent experimental and theoretical developments suggest that possible excitations of the core may affect not only the structure but also the reaction dynamics. In these situations, the structure of the projectile is better described in terms of a particle-plus-core model, in which several states of the core are included explicitly.

In a recent work [1] we have generalized the Transformed Harmonic Oscillator (THO) basis in order to include the excitations of the core in a simple particle-rotor model. Diagonalizing the Hamiltonian in a square-integrable basis, the eigenstates obtained or pseudo-states (PS) are a finite approximation of the bound and unbound states of the nucleus. The THO basis has been recently used

\footnotetext{
ae-mail: lay@pd.infn.it
} 
within this extended DWBA framework, referred to hereafter as DWBAx, in order to analyse resonant break-up of halo nuclei [2]. In this contribution, we apply the DWBAx method to describe the resonant break-up of ${ }^{11} \mathrm{Be}$ on ${ }^{12} \mathrm{C}$ at $67 \mathrm{MeV} / \mathrm{u}$, and discuss the interplay between the valence and core excitation mechanisms in the break-up angular distributions. We will also discuss the possibility of extracting spectroscopic information through this interference between both excitation mechanisms shown in [2].

\section{Particle-plus-core model in a pseudo-state basis}

In the weak-coupling limit, the Hamiltonian of a core+valence system is written in the form

$$
H=T_{r}+V_{v c}(\vec{r}, \xi)+h_{\text {core }}(\xi),
$$

where $h_{\text {core }}(\xi)$ represents the internal Hamiltonian of the core, with eigenstates $\phi_{I}(\xi) . V_{v c}(\vec{r}, \xi)$ is the interaction between the particle and the core and it is responsible for the coupling between the valence particle motion and the excitations of the core. It depends on the model assumed for the excitations of the core. For example, in a particle-rotor model, we assume the core to be permanently deformed. In this case, the interaction with the valence particle would depend on the state of rotation of the deformed core and the relative orientation between particle and rotor.

If the coupling is weak we can consider the total wavefunction as a combination of core states and valence configurations coupled to the corresponding total angular momentum $J$ :

$$
\Psi_{\varepsilon ; J M}(\vec{r}, \xi)=\sum_{\alpha} R_{\varepsilon, \alpha}^{J}(r)\left[\mathcal{y}_{\ell s j}(\hat{r}) \otimes \phi_{I}(\xi)\right]_{J M},
$$

where $\mathcal{Y}_{\ell s j m}(\hat{r})=\left[Y_{\ell}(\hat{r}) \otimes \chi_{s}\right]_{j m}$, with $\chi_{s}$ a spin function, and $\ell$ the orbital angular momenta. Each term of this sum is called channel and it is characterized by a given set of quantum numbers $\alpha=$ $\{\ell, s, j, I\}$. For each channel we construct a THO set of functions. We then diagonalize the Hamiltonian so that the final PS will be a linear combination of the corresponding THO functions [1].

\section{Core excitations in resonant break-up}

The contribution to the break-up cross section for a given channel $f$ can be expressed in terms of the transition matrix $\mathcal{T}$ :

$$
\frac{d \sigma_{f}}{d \Omega}=\frac{1}{(2 J+1)} \frac{\mu_{p t}}{\left(2 \pi \hbar^{2}\right)^{2}} \frac{k_{f}}{k_{i}} \sum_{M M^{\prime}}\left|\mathcal{T}^{J M J^{\prime} M^{\prime}}\right|^{2},
$$

where $\mu_{p t}$ is the reduced mass between projectile and target, and $k_{i}$ and $k_{f}$ are the initial and final linear momenta.

In the DWBA approximation, the $\mathcal{T}$ matrix is given by the integral:

$$
\mathcal{T}^{J M J^{\prime} M^{\prime}}=\left\langle\widetilde{\chi}_{\vec{k}_{f}}^{(-)}(\vec{R}) \Psi_{f ; J^{\prime} M^{\prime}}(\vec{r}, \xi)\left|V_{p t}\right| \Psi_{i ; J M}(\vec{r}, \xi) \widetilde{\chi}_{\vec{k}_{i}}^{(+)}(\vec{R})\right\rangle,
$$

where $\Psi_{i ; J M}$ and $\Psi_{f ; J^{\prime} M^{\prime}}$ are the initial and final wavefuncitons for the projectile. $\widetilde{\chi}_{\vec{k}_{i}}^{(-)}$and $\widetilde{\chi}_{\vec{k}_{f}}^{(+)}$are the projectile-target distorted waves, solutions of the corresponding homogeneous equations.

According to the previous section, the wavefunctions will be defined as (2). The projectile-target potential $V_{p t}$, sum of valence particle-target and core-target potentials, will depend on the internal degrees of freedom of the core:

$$
V_{p t}=V_{v t}\left(R_{v t}\right)+V_{c t}\left(\vec{R}_{c t}, \xi\right)
$$


The second term is conveniently expanded in multipoles as $V_{c t}^{\lambda=0}\left(R_{c t}\right)+\sum_{\lambda \mu} V_{c t}^{\lambda>0}\left(R_{c t}, \xi\right) Y_{\lambda \mu}(\hat{\xi})$. If we add the $\lambda=0$ multipole to the valence-target interaction, we recover the $\mathcal{T}$ matrix corresponding to the case without core excitations. Therefore, the $\mathcal{T}$ matrix can be split into two parts:

$$
\mathcal{T}^{J M J^{\prime} M^{\prime}}=\mathcal{T}_{\text {val }}^{J M J^{\prime} M^{\prime}}+\mathcal{T}_{\text {corex }}^{J M J^{\prime} M^{\prime}}
$$

When the recoil of the core nucleus can be neglected, $\vec{R}_{b t} \approx \vec{R}$, the second part $\mathcal{T}_{\text {corex }}^{J M J^{\prime} M^{\prime}}$ acquires a very simple form which can be related to the inelastic scattering of the core on the target [3]. The total cross section will be the coherent sum of both components, and so interference effects will occur. A more detailed description can be found in [3].

\section{Application to ${ }^{11} \mathrm{Be}+{ }^{12} \mathrm{C}$ reaction}

We have studied the break-up of ${ }^{11} \mathrm{Be}$ on ${ }^{12} \mathrm{C}$ at $67 \mathrm{MeV} / \mathrm{u}$ using the DWBAx reaction framework to describe the break-up process and the THO method to describe the ${ }^{11} \mathrm{Be}$ continuum. We compare the results of the calculations with the experimental data from RIKEN [4]. The experimental break-up energy distribution shows two bumps associated to the two first positive parity low-lying resonances in ${ }^{11} \mathrm{Be}$. In figure 1 , we show the angular distributions of the break-up cross section for the two bumps $\left(5 / 2^{+}\right.$and $\left.3 / 2^{+}\right)$. We compare them with the DWBAx calculation for the first $5 / 2^{+}$and $3 / 2^{+}$resonances.

If we use the particle-rotor model (PRM) with the Hamiltonian of Ref. [5] (model Be12-b) we obtain the following spectroscopic decomposition for the ground state and the the first $5 / 2^{+}$and $3 / 2^{+}$ resonances [1]:

$$
\begin{array}{ll}
\left|\Psi_{\text {g.s. }}\right\rangle= & \sqrt{0.86}\left|0^{+} \otimes s_{1 / 2}\right\rangle \quad+\sqrt{0.02}\left|2^{+} \otimes d_{3 / 2}\right\rangle+\sqrt{0.12}\left|2^{+} \otimes d_{5 / 2}\right\rangle \\
\left|\Psi_{5 / 2^{+}}\right\rangle= & \sqrt{0.70}\left|0^{+} \otimes d_{5 / 2}\right\rangle+\sqrt{0.18}\left|2^{+} \otimes s_{1 / 2}\right\rangle+\sqrt{0.01}\left|2^{+} \otimes d_{3 / 2}\right\rangle+\sqrt{0.11}\left|2^{+} \otimes d_{5 / 2}\right\rangle \\
\left|\Psi_{3 / 2^{+}}\right\rangle= & \sqrt{0.16}\left|0^{+} \otimes d_{3 / 2}\right\rangle+\sqrt{0.74}\left|2^{+} \otimes s_{1 / 2}\right\rangle+\sqrt{0.02}\left|2^{+} \otimes d_{3 / 2}\right\rangle+\sqrt{0.08}\left|2^{+} \otimes d_{5 / 2}\right\rangle .
\end{array}
$$

In Ref. [2], DWBAx calculations were performed for the same data using these wavefunctions. A good agreement was found in shape, although the magnitude was overestimated. Here, in order to explore the possibility of extracting spectroscopic information from the experimental data, we propose a simplified model wavefunction for each resonance of the form:

$$
\begin{aligned}
& \left|\Psi_{5 / 2^{+}}\right\rangle=a\left|0^{+} \otimes d_{5 / 2}\right\rangle+b\left|2^{+} \otimes s_{1 / 2}\right\rangle+\left(1-a^{2}-b^{2}\right)^{1 / 2}\left|2^{+} \otimes d_{5 / 2}\right\rangle \\
& \left|\Psi_{3 / 2^{+}}\right\rangle=c\left|0^{+} \otimes d_{3 / 2}\right\rangle+d\left|2^{+} \otimes s_{1 / 2}\right\rangle+\left(1-c^{2}-d^{2}\right)^{1 / 2}\left|2^{+} \otimes d_{5 / 2}\right\rangle,
\end{aligned}
$$

keeping the radial shape from the PRM calculation and adjusting the parameters $a, b$ (for the $5 / 2^{+}$ resonance) and $c, d$ (for the $3 / 2^{+}$resonance) to minimize the $\chi^{2}$ value. We allow also for a global normalization factor $(\mathrm{N})$, so we search for the resonant wavefuntions that best-fit the shape of the experimental data, irrespective of the normalization. In order to estimate the error bars, we look for an increase of 1 in the $\chi^{2} / n$ value, being $n$ the number of experimental points. The results will also depend on the ground state wavefunction, for which we adopt that from the PRM calculation [1].

Following this prescription, we find for the $5 / 2^{+}$resonance the coefficients $a^{2}=0.55 \pm 0.15$, $b^{2}=0.30 \pm 0.15$ and a renormalization factor $\mathrm{N}=0.50 \pm 0.04$. The need for this renormalization could reflect the presence of additional components in the ground state or resonant wavefunctions, not considered in our model wavefunctions, but also uncertainties in the absolute normalization of the data. The weight of the component corresponding to the ${ }^{10} \mathrm{Be}$ ground state is somewhat smaller, but consistent with the particle rotor and Shell Model predictions, see table 1 in [2]. For the $3 / 2^{+}$ resonance we obtain $c^{2}=0.40 \pm 0.20, d^{2}=0.55 \pm 0.20$ and $\mathrm{N}=0.31 \pm 0.03$. For this resonance, there 
is a smaller sensitivity to the spectroscopic factors but, again, the extracted values are consistent with the theoretical ones. The fitted cross sections are included in figure 1. These cross sections are similar to those shown in [2] due to the fact that the original spectroscopic factors are consistent with those obtained from the fit. We can also see in figure 1 that the valence contribution is dominant for the $5 / 2^{+}$ resonance whereas, in the $3 / 2^{+}$case, the main contribution comes from core excitations, as expected in view of the main spectroscopic factors obtained from the fit.
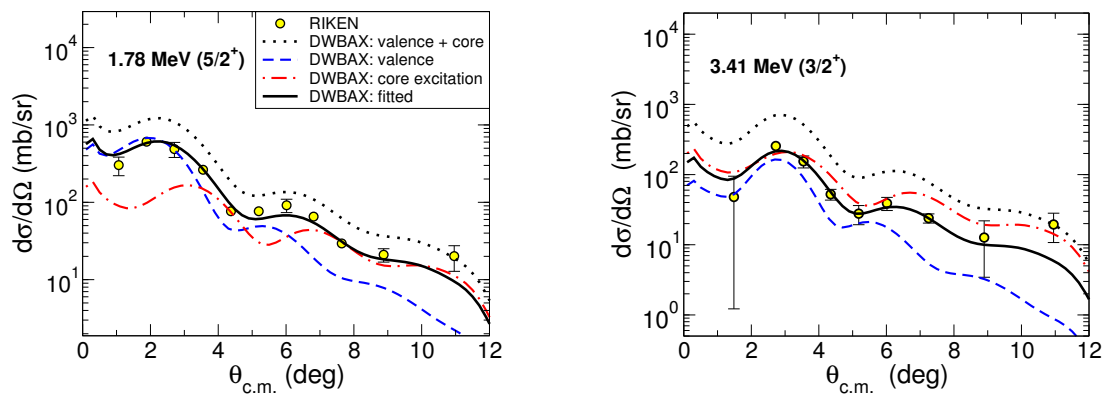

Figure 1. Valence (dashed line) and core (dot-dashed line) contributions to the break-up of the 1.78 and 3.41 $\mathrm{MeV}$ resonances populated in the ${ }^{11} \mathrm{Be}+{ }^{12} \mathrm{C}$ reaction at $67 \mathrm{MeV} / \mathrm{u}$, using a particle-core description of the ${ }^{11} \mathrm{Be}$ nucleus and fitting the spectroscopic factors as explained in the text. The dotted line is the coherent sum of both contributions. The solid line shows the total cross section once we renormalize the calculation by the global normalization factor obtained from the $\chi^{2}$ analysis.

\section{Conclusions}

We have shown that the PS method with THO functions provides a suitable discrete description of the continuum of a two-body system including possible excitations of the core. It provides a natural treatment of resonances, which simplifies the analysis of resonant break-up. The break-up cross sections obtained from DWBAx calculations show sensitivity to the spectroscopic factors. It is possible to extract values of the spectroscopic factors by fitting the angular distributions of the resonant break-up data. The method has been applied to the ${ }^{11} \mathrm{Be}+{ }^{12} \mathrm{C}$ reaction at $67 \mathrm{MeV} / \mathrm{u}$, for which experimental data exist [4]. Assuming that the ground state wavefunction of ${ }^{11} \mathrm{Be}$ is well described by that calculated in the particle-rotor model, the spectroscopic factors for the main components of the resonant states have been extracted, and found to be in good agreement with particle-rotor and shell-model predictions. These results encourage the application of this method to other weakly-bound systems with deformed core like ${ }^{17} \mathrm{C}$ or ${ }^{19} \mathrm{C}$.

In conclusion, the analysis presented here propose a useful method to extract spectroscopic information for those halo nuclei that can be understood as two-body systems with a non inert core. Resonances mainly based on excited states of the core are difficult to populate in other reactions like transfer. The analysis of resonant break-up data, like the one presented here, provide an alternative way to obtain the spectroscopic factors for these resonances [2].

\section{References}

[1] J.A. Lay, A.M. Moro, J.M. Arias, J. Gómez-Camacho, Phys. Rev. C 85, 054618 (2012)

[2] A.M. Moro, J.A. Lay, Phys. Rev. Lett. 109, 232502 (2012)

[3] A.M. Moro, R. Crespo, Phys. Rev. C 85, 054613 (2012)

[4] N. Fukuda et al., Phys. Rev. C 70, 054606 (2004)

[5] F.M. Nunes et al., Nucl. Phys. A 609, 43 (1996) 